\title{
A case of splenic artery pseudoaneurysm rupture in a bulimic patient with gastric ulcer
}

\author{
Lucio Brugioni, ${ }^{1}$ Jolanda Petri, ${ }^{2}$ Silvia Speranza Cirino, ${ }^{2}$ Lucia Amidei, ${ }^{2}$ Serena Scarabottini, ${ }^{2}$ \\ Francesca De Niederhausern, ${ }^{1}$ Daniela Vivoli, ${ }^{1}$ Massimo Girardis ${ }^{3}$ \\ ${ }^{1}$ Internal Medicine and Critical Area Unit Critica Unit, Policlinico Modena; ${ }^{2}$ Emergency Medicine, University of Modena and \\ Reggio Emilia; ${ }^{3}$ Intensive Care Unit, Policlinico Modena, Italy
}

\begin{abstract}
Splenic artery pseudoaneurysm is a rarely described condition and it is even more rarely considered as a complication of peptic disease. The most common etiologies are pancreatitis, both chronic and acute, and trauma. Diagnosis can be challenging, including ultrasonography, computed tomography scan and angiography, the latter being useful also for therapy (embolization). Given her history of bulimia nervosa and the stress related to hospitalization, our patient was particularly predisposed to peptic ulcer.
\end{abstract}

\section{Introduction}

Purging behavior including self-induced vomiting, diuretics and laxative abuse can lead to severe medical and surgical complications. We present a case of a bulimic patient who most likely developed a rare complication (splenic artery pseudo aneurysm rupture) of a more common condition (peptic gastric ulceration) in the context of a complex clinical scenario.

\section{Case Report}

A 51-year-old female patient with long-standing bulimia nervosa came to the emergency department with abdominal pain and hypovolemic shock secondary to Senna laxative abuse. Plain radiography of the abdomen was obtained and showed air-fluid levels in the transverse colon with decreased haustra

Correspondence: Lucio Brugioni, Internal Medicine and Critical Area Unit, Policlinico di Modena, via del pozzo 71, 41121 Modena, Italy.

Tel.: +39.059.4225491. E-mail: luciob2362@gmail.com

Key words: Splenic artery pseudoaneurysm; peptic ulcer; bulimic disorder.

Conflict of interests: the authors declare no potential conflict of interests.

Received for publication: 1 December 2019.

Accepted for publication: 14 January 2020.

This work is licensed under a Creative Commons Attribution NonCommercial 4.0 License (CC BY-NC 4.0).

${ }^{\circ}$ Copyright: the Author(s), 2020

Licensee PAGEPress, Italy

Italian Journal of Medicine 2020; 14:100-101

doi:10.4081/itjm.2020.1244 and air and feces inside the rectum. Laboratory tests revealed metabolic acidosis, hyponatremia, leukocytosis and normal c-reactive protein.

Suspecting an ischemic/toxic bowel inflammation, supported by the consult of Anti-Poisoning Centre specialists, she was administered activated charcoal and IV hydration therapy. She was admitted to a SubIntensive department and then transferred to an Intensive Care Unit after the execution of abdomen computed tomographic (CT) scan, which confirmed colonic edema without vascular etiology.

After one week of supportive care therapy she developed melena due to large ulceration in the stomach fundus, without any active bleeding, for which protonpump inhibitors (PPI) therapy was initiated. She remained stable for about ten days, then she had an abrupt cardiac arrest with subsequent return of spontaneous circulation after cardiopulmonary resuscitation maneuvers. The hypothesis of hemorrhagic etiology was made discovering a rapid decrease of hemoglobin levels $(3 \mathrm{gr} / \mathrm{dL})$. With this suspicion an emergency gastroscopy was performed: the previously described ulceration was only partially visualized because of clots and active bleeding, which was not susceptible of local therapy. The emergency angiography revealed a massive splenic artery lesion bleeding through the ulceration inside the stomach lumen. The pseudo aneurysm was then embolized as a life-saving treatment. Further complications occurred, like post-anoxic coma, pulmonary embolism and pneumonia; the patient had a second cardiac arrest that turned out to be fatal.

\section{Discussion}

Laxative abuse is the second most frequent purging behavior in bulimia nervosa, after self-induced 
vomiting. ${ }^{1,2}$ Gastrointestinal complications include melanosis coli, cathartic colon, and functional impairment, which can lead to hypovolemia and electrolyte disturbances. Esophagitis, gastroesophageal reflux and gastropathy are also common findings in patients with eating disorder., ${ }^{1,2}$

Stress ulcer in critically ill patients is a known entity and because of this evidence PPI prophylaxis is often administered, even though, given the possibility of adverse effects of acid suppression, even high-risk patients may not achieve any clear benefit from this treatment. ${ }^{3}$

Splenic artery pseudo aneurysm is a rare entity caused mostly by pancreatitis (acute and chronic) and trauma (including iatrogenic causes), while the association with peptic disease is uniquely cited in a few case reports, only three up to 2003 as reported by Tessier et al. ${ }^{4}$ and other four up to $2018 .^{5-8}$ Just like in pancreatitis, the arterial walls weakening is attributed to digestion of the splenic artery by pancreatic enzymes, probably gastric acid plays the same role on the vessel layers, leading to pseudo aneurysm formation. Presentation of splenic artery pseudo aneurysm varies from incidental finding to acute hemodynamic shock and, considering the rarity of this condition, diagnosis can be challenging. CT and ultrasonography are the most used diagnostic methods, but small pseudo aneurysm may be missed; angiography is the most reliable technique and offers the possibility to be therapeutic in patients who can benefit from embolization. Surgery treatments include splenectomy with or without distal pancreatectomy and ligation of the artery. Since symptoms and risk of rupture do not correlate with size, repair of all pseudo aneurysm is suggested.

\section{Conclusions}

From this patient case, given the bulimic disorder history and the stress of hospitalization, both correlated with the possibility of developing gastric ulcer, we should take into consideration the opportunity of early gastric protection. Splenic artery pseudo aneurysm is a rare condition and in case of hemorrhagic shock, it may be difficult to think about it in a list of more common differential diagnoses. We suggest that peptic ulcer may considered as a rare etiology for splenic artery pseudo aneurysm.

\section{References}

1. Mehler PS, Rylander M. Bulimia Nervosa-medical complications. J Eating Disord 2015;3:12.

2. Forney KJ, Buchman-Schmitt JM, Keel PK, Frank GK. The medical complications associated with purging. Int J Eating Disord 2016;49:249-59.

3. Cook D, Guyatt G. Prophylaxis against upper gastrointestinal bleeding in hospitalized patients. N Engl J Med 2018;378:2506-16.

4. Tessier DJ, Stone WM, Fowl RJ, et al. Clinical features and management of splenic artery pseudoaneurysm: case series and cumulative review of literature. J Vasc Surg 2003;38:969-74.

5. Syed SM, Moradian S, Ahmed M, et al. A benign gastric ulcer eroding into a splenic artery pseudoaneurysm presenting as a massive upper gastrointestinal bleed. J Surg Case Rep 2014;2014:11.

6. Sawicki M, Marlicz W, Czapla N, et al. Massive upper gastrointestinal bleeding from a splenic artery pseudoaneurysm caused by a penetrating gastric ulcer: case report and review of literature. Polish J Radiol 2015;80:384.

7. Haivas CD, Kessler S. Splenic artery pseudoaneurysm causing brisk upper-GI bleed from gastric ulcer. Gastroint Endoscopy 2016;84:540-1.

8. Cho SB, Park SE, Lee CM, et al. Splenic artery pseudoaneurysm with splenic infarction induced by a benign gastric ulcer: A case report. Medicine 2018;97:29. 